\title{
Maintenance process modelling with Cellular Automata and Voronoi diagram
}

\author{
M. Ouardouz ${ }^{1}$, M. Kharbach ${ }^{1}$, Bel Fekih ${ }^{1}$, A. Bernoussi ${ }^{2}$ \\ ${ }^{I} M M C,{ }^{2}$ GAT Faculty of Sciences and Techniques, B.P.416, Tangier, Morocco
}

\begin{abstract}
Resource allocation problems in industrial processes have been widely studied. In this paper we present a case of maintenance technicians' allocation process simulation using cellular Automata and Voronoi diagram taking into account the spatial constraints. The aim is to extend the spectrum of constraints of the resource allocation problems and improve the previous works by carrying out the spatial dimension impact on performance optimization. To illustrate our approach some simulations are given.
\end{abstract}

Keywords: Cellular Automata, resource allocation, industrial, maintenance, Vornoi diagram

\section{Introduction}

In the manufacturing industries, many approaches are increasingly evolved in new organizational forms in the aim to optimize the performance by reducing costs, time as well as quality improvement. The optimization of human resources allocation and the efficient use of their competencies emerge as key points of competitiveness of such organizations [1], [2]. The resources allocation is justified by various researches that showed that despite automation, ever higher, of production systems of a company, the human entity remained one of the key points of its success and sustainability facing the rapidly changing in the business environments $([3,4])$.

Several mathematical models have been developed $[5,6]$. These models treat the system considering the human resources allocation without taking into account, explicitly, the spatial dimension. They focus, especially, on the integration of competencies, flexibility and / or versatility in allocation, planning or scheduling problems.

The combined integration of competencies of technicians and their displacement in the workshop (space frame) in resource allocation problems are rarely discussed in literature. However, existing works concerns, essentially, resource planning (nurses) in the hospital sector, for example, but define competencies as the difference between human resources in terms of grades or qualifications [7]. The works of Bennouri [1], have claim to incorporate the individual and collective competencies factor as a key process efficiency issue.

All these works attach particular importance to the involvement of competencies as a distinctive element of performance and propose to quantify the impact of individual and collective dimensions of these competencies. Moreover, given that the allocation depends heavily on the geographic area (factory, city, country), the spatial dimension cannot be overlooked in the allocation models.

The complexity of the problem increase by the introduction of the spatial variable, that's why we opted for the use of Cellular Automata (ACs)[8] to approach the problem. The ACs through simple rules can model and simulate complex systems [9]. The implementation of such model in an industrial context taking in account spatiotemporal constraints has not yet been studied satisfactorily.

The approach presented in this article comes under the optimization under constrains and decisionaiding using cellular automata (CA). This tool will contribute to integrate the spatial dimension in the resource allocation process and to simulate its evolution. The objective of this paper is to develop a general approach of optimization and visual simulation by cellular automata based on the integration of spatiotemporal factors in a resources allocations problem (Location of equipments and facilities, technician displacements)

The studied problem is the particular case of a manufacturing company consisting of several equipments and facilities, geographically distributed, subject to wide kinds of maintenance interventions requiring different competencies. The paper concerns the assignment of interventions of preventive maintenance tasks.

\section{Cellular Automata Principle And Problem Statement}

Consider a set $\mathcal{E}$ of equipment in a domain space $\Omega \subset R^{n}, n=2$, and a set $\mathcal{P}$ of technicians. Maintenance should meet the demand of different departments of the company by improving the availability of equipments and facilities. The number of interventions that can be processed simultaneously depend on the technician's skills level and on their competencies. The response time is conditioned by the choice of the resource and its proximity to the equipment or installation. However, all technicians must be engaged (busy) in a uniform manner, to avoid work overload and optimize transport and travel. The problem statement is given with 
a cellular automaton approach as follow:

1.1. Cellular Automata Principle

Cellular Automata (CA) are a class of spatially and temporally discrete mathematical systems characterized by local interaction and synchronous dynamical evolution. CAs were proposed by Von Neumann and Ulam in the early $1950 \mathrm{~s}$ as models for self-replicating systems [10]. Since then, CA properties have been widely investigated, and CAs have been applied to simulating and studying phenomena in complex systems, in such diverse - fields as physical, biological, and social systems. A standard CA consists of a regular infinite uniform lattice (the infinite space $\mathbb{Z}^{d}$, where $d$ is the space dimension.

Generally used, otherwise, a finite set $\prod_{i=1}^{i=d}\left\{0,1, \cdots, N_{i}\right\}$ which captures periodicity of period $N_{i}$ in spatial dimension $i$ is considered) with a discrete variable at each site, named cell. The state of each cell is updated based on the previous states of its immediate neighbouring cells according to a set of local rules. In what follows, the time variable is given in $I=\{0,1,2, \cdots\}$.

Definition:a cellular automaton is a quadruple $\mathcal{A}=(\mathcal{L}, \mathcal{S}, N, f)$ where:

- $\mathcal{L}$ is a lattice defined by a regular grid of cells denoted by $c_{i_{1} i_{2} \cdots i_{d}}$, on a domain $\Omega$,

- $\mathcal{S}$ a finite set of states given as a commutative ring $\mathbf{S}=\{0,1, \cdots, k-1\}$ in which modular arithmetic will be used,

- $\quad N$ a neighbourhood of the cell $c$,

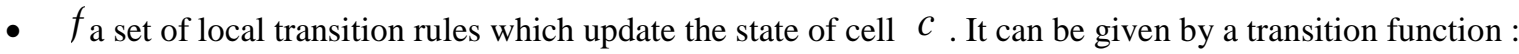

$$
f: \begin{array}{ll}
\mathcal{S}^{N(c)} & \rightarrow \mathcal{S} \\
s_{t}(N(c)) & \rightarrow s_{t+1}(c)
\end{array}
$$

The study of CA as a dynamical system which involves the analysis of phase space, leads to the so-called global CA state or CA configuration defined as a function from $\mathcal{L}$ to $\mathcal{S}$ which maps cells to states. The CA configuration at time $t$, denoted by $s_{t}$ is given as an element of $E=\mathcal{S}^{\mathcal{L}}$, the state space which covers all of the states in which a system could possibly be in.

The CA state evolution obeys to the so-called global dynamics defined by

$$
\begin{aligned}
F: E & \rightarrow E \\
s_{t} & \rightarrow s_{t+1}
\end{aligned}
$$

\subsection{Problem statement}

To apply the cellular automata approach to our resource allocation problem, we start by defining the corresponding cellular automaton $\mathcal{A}=(\mathcal{L}, \mathcal{S}, N, f)$.

- The lattice $\mathcal{L}$ corresponds to a grid of an bidimentional space $(d=2)$ such as $: \mathbf{L}=\left\{\begin{array}{l}c_{i j}, \\ 1 \leq i \leq N \\ 1 \leq j \leq M\end{array}\right\}$ where $N \times M$ represents the total number of cells $\mathcal{L}$. We assume that our domain is bounded.

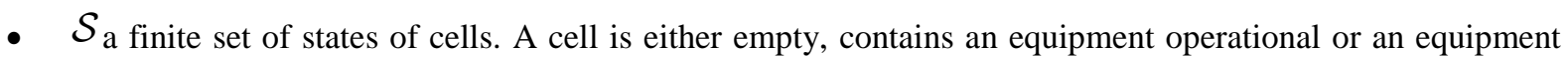
down or an assigned for maintenance or available technician, or busy one or assigned one:

Equipments, are indexed in a set $\mathcal{E}, i=\{1, \ldots, E\}$ and technicians in a set $\mathcal{P}=\{1, \cdots, P\}$.

o The equipment, noted $\mathcal{E}_{m}$ for $m \in \mathcal{E}$, can be Operational or Down. We assign to each state the values :

$\theta\left[E_{m}\right]= \begin{cases}1 & \text { equipment } E_{m} \text { operationd } \\ -1 & \text { down } \\ -0.5 & \text { is assignedto a technician }\end{cases}$ 
o Each equipment $\mathcal{E}_{m}$ is characterized by its mean time between failures noted $M T B F_{j}$. and its mean time to repair noted $M T T R_{j}$

o The technician, noted $P_{k}$ for $k \in \mathcal{P}$, has a state $\varphi\left[P_{k}\right]$ defined by: $\varphi\left[P_{k}\right]=\left\{\begin{array}{cc}2 & \text { technician } P_{k} \text { available } \\ -2 & P_{k} \text { is assignedto an equipment } E_{m}\end{array}\right.$

Then the state space is given by:

$\mathbf{S}=\{-2,-1,-0.5,0,1,2\}$

where the state of each cell is :

o $\quad e\left(c_{i j}\right)=-1$, if the cell is occupied by a down equipment ;

o $\quad e\left(c_{i j}\right)=-0.5$, if the cell is occupied by an equipment with an assigned technician ;

o $\quad e\left(c_{i j}\right)=1$ if the cell is occupied by an operational equipment ;

o $\quad e\left(c_{i j}\right)=2$ if the cell is occupied by an available technician ;

o $\quad e\left(c_{i j}\right)=-2$ if the cell is occupied by an assigned technician ;

o $\quad e\left(c_{i j}\right)=0_{\text {if the cell is empty. }}$

- $\quad$ Neighbourhood: In the case of cellular automata defined on Euclidean grids $\mathcal{L}=\mathbb{Z}^{d}$ where cells are given by their $d$ integer coordinates, two classes of neighbourhoods are classically chosen, the von Neumann neighbourhoods and the Moore neighbourhoods. They are characterized by a radius $r$ and defined by:

$$
N(c)=\left\{c^{\prime} \in \mathcal{L} \mid\left\|c^{\prime}-c\right\|_{i} \leq r\right\}
$$

where $\|c\|_{i}, i=1, \infty$ indicates the sum and the maximum respectively, of the absolute value of the components of cell $c$.

- Transition rules: The evolution of the state of each cell $c_{i j}$, depends on its state and those of its neighbourhood. They are summarized as follow:

$$
\begin{aligned}
& e_{t}\left(c_{i j}\right) \rightarrow e_{t+1}\left(c_{i j}\right) \\
& \text { if } \quad e_{t_{i}}\left(c_{i j}\right)=0 \text { then } e_{t_{i+1}}\left(c_{i j}\right)=\left\{\begin{array}{cc}
2 & \text { if it is occupaiedby an availabletechnician } \\
-1 & \text { if we installan equipement } \\
-2 & \text { if it's occupeiedby an assignedtechnician } \\
0 & \text { otherwise }
\end{array}\right. \\
& \text { if } \quad e_{t_{i}}\left(c_{i j}\right)=1 \text { then } e_{t_{i+1}}\left(c_{i j}\right)=\left\{\begin{array}{cc}
1 & \text { if the equipementcontinu towork } \\
-1 & \text { if the equipementstopwork } \\
0 & \text { sion retire l'equipement }
\end{array}\right. \\
& \text { if } e_{t_{i}}\left(c_{i j}\right)=-1 \text { then } e_{t_{i+1}}\left(c_{i j}\right)=\left\{\begin{array}{cc}
-0.5 & \text { if there exists an assaignedtechniciant } \\
-1 & \text { if thereis no assignedtechniciant } \\
0 & \text { sion retire l'équipement }
\end{array}\right. \\
& \text { if } e_{t_{i}}\left(c_{i j}\right)=-0.5 \text { then } e_{i+1}\left(c_{i j}\right)=\left\{\begin{array}{cc}
-0.5 & \text { Si pasencoreréparée } \\
1 & \text { Si machine réparée }
\end{array}\right.
\end{aligned}
$$

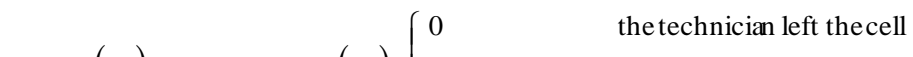

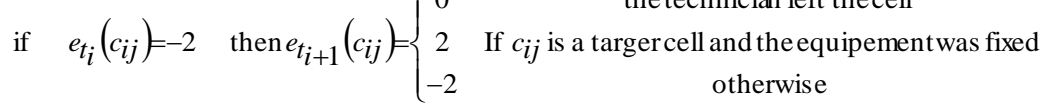

$$
\begin{aligned}
& \text { if } e_{t_{i}}\left(c_{i j}\right)=2 \text { then } e_{t_{i+1}}\left(c_{i j}\right)=\left\{\begin{array}{cc}
0 & \text { if the technician move } \\
-2 & \text { if } c_{i j} \text { is a targetcell } \\
2 & \text { if the technician didn't move }
\end{array}\right.
\end{aligned}
$$




\section{ProblemStatement:}

The problem consist keeping all (or the maximum) equipments operational and if some intervention of technicians are needed, to do this with an optimal way: minimal cost, energy, and displacements... etc; In this work we consider this problem through the technicians displacements.

Remark: The state evolution depends on the technicians' displacement. Indeed, the technicians are moving toward the down equipments to fix them. We will consider two cases:

1.The case where the technician must move to the nearest down equipment (autonomous system);

2. The case where the assignments of the technicians are ordered by a master schedule (controlled case). This schedule is an active control applied to the CA model. For example the daily schedule of the technicians gives for each one a list of interventions to be performed and specifies the cell to reach. If a new equipment failure is reported, the schedule will be updated and new assignments will be generated based on the availability of technicians and/or their proximity to equipment. In this paper we will not consider the controlled case and this problem will be studied in a further paper.

We consider the first case (autonomous system) but with optimal displacements of the technicians. We associate to each equipment, operational or down, an empty cell, named target cell, it's the cell that the technicians have to reach in order to fix the equipment (fig1).

1.3. Technician displacements: principle

The technician moves if there's a down equipment to repair it otherwise he goes back to the office. The principal is explained in the following algorithm:

Considering the position (cell $c_{i j}$ ) of the technician:

1. For $r=1$, in case there's unique equipment down in the neighbourhood of diameter $r$, then the technician have to move to it ;

otherwise, if there's more than one down equipment we will the Voronoi diagram else if $r<R$, take $r=r+1$ and return to 1 ; else if $r=R$ (which mean that all equipments are operational), return to case technician. where $R$ is the maximum diameter of the neighbourhood $\mathcal{N}$ such that $\mathcal{N} \subset \mathcal{T}$.

To simplify the model, the technicians will move only north, south, east or west, so there will be no displacement along the diagonal cells.

For simulation we have to consider initial conditions, with that conditions there'smore than one solution for the displacement of the technicians. To overcame this problem we will consider an optimal displacement of the technician in the sense to minimize the trajectory of each technician (and consequently for all technicians). Technician displacements

To optimize the technician's displacements we consider an algorithm in two steeps.

1. First, to assign technician to equipment, we consider a Voronoi diagram for both the down equipments and available technicians

2. In each Voronoi cell we consider an "optimal displacement". For that we assume that the displacements are performed in a chosen direction depending on the technician occupying cell $c_{i j}$.

We recall in the following subsection the Voronoi diagram definition [3]

Voronoi diagram generalities:

Consider the set of points $\left\{P_{1}, P_{2}, \ldots, P_{p}\right\}$ in a domain $\Omega \subset R^{2}$..

For each point $P_{k}$ we determinate the set:

$\mathbf{R}_{k}=\left\{X \in \Omega: \quad d\left(X, P_{k}\right) \leq d\left(X, P_{j}\right) \quad \forall j \neq k\right\}$

where $d\left(X, P_{k}\right)$ is a distance from $X$ to $P_{k}$.

The set $\mathcal{R}_{k}$ is called Voronoi cell with seed (site, or generator) $P_{k}$. It is the region of all points closer to that seed than to any other.

The Voronoi diagram is the union of all the Voronoi sells:

$$
\operatorname{Vor}\left(\left(P_{k}\right)_{1 \leq k \leq p}\right)=\bigcup_{1 \leq k \leq p} \mathbf{R}_{k}
$$

For more details see [2]. 
Algorithm: we use the Voronoi diagram as follow:

1- We consider the Voronoi diagram $\operatorname{Vor}(E d)=\bigcup_{1 \leq k \leq p^{\mathrm{R}}}^{E}$ associated to the set of down equipments

$\mathcal{E}_{d}=\left\{E_{j}, 1<j<E, \operatorname{phi}\left(E_{j}\right)=-1\right\}$.

- If in each Voronoi cell there's a unique unavailable technician then the technician is assigned to the equipment;

- Else if the Voronoi cell contains more than one available technician then, we consider a new Voronoi diagram, $\operatorname{Vor}(T a)=\bigcup_{1 \leq k \leq p} \mathcal{R}_{k}^{T}$ relatively to the set $T a$ of those available technicians and the technician is assigned to the equipment in the intersection between the two Voronoi cells $\mathbf{R}_{k}^{E} \cap \mathbf{R}_{k}^{T} \neq$ ?

- Else if the equipments Voronoi cell contains no technician then continue 2- We eliminate both assigned technicians and equipments and return to 1

Remark:ifequipment is located equidistant to two technicians or a technician is located equidistant to two equipments we apply a right priority rule. This algorithm is illustrated in the example figure 1. below:
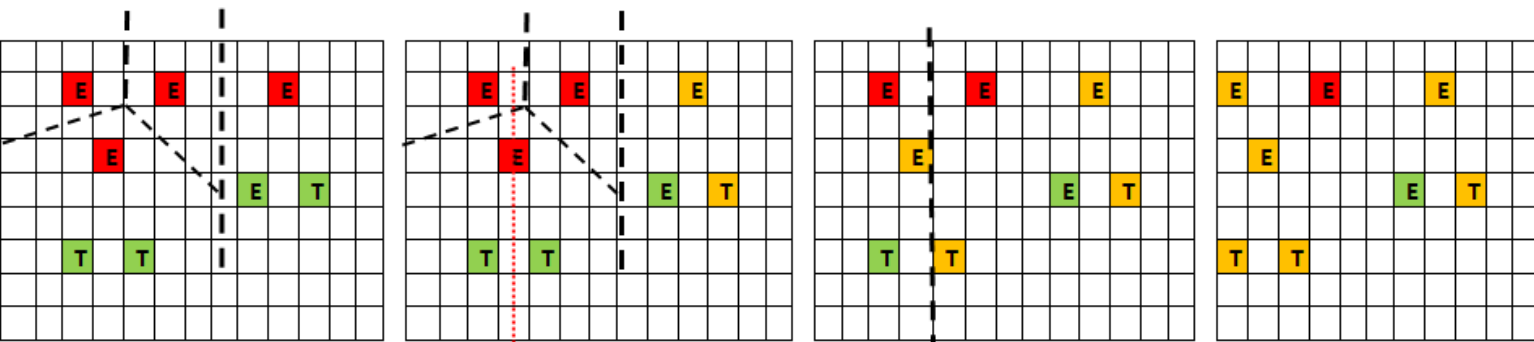

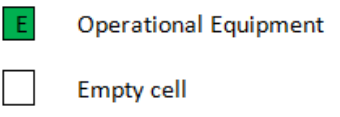

E Down Equipment
$\mathrm{T}$ Available Technician

E Assigned Equipment

Fig.1.Allocation by Voronoi diagram

Gridl in figure 1: Voronoi cells are created using down machines as seeds and then a technician is assigned to the machine if he is in the Voronoi domain of that down machine

Grid2 in figurel:In case there's more than one technician a second Voronoi diagram is created using the technician as seeds after that we assign to the down equipment the technician in the intersection of the two Voronoi domains.

Grid3 in figure1: we eliminate the assigned machine and we draw a new Voronoi diagram

This guarantees an optimal allocation of the technician to the machines.

\section{Displacement rules:}

Respect to the Voronoi diagram, the following traffic rules are adopted:

- If two technicians cross in perpendicular directions: the rule of the priority to the right will be adopted.

- If two technicians target the same empty cell with opposite directions, we perform, for at least one of them, a $\pi / 2$ rotation to the right of its direction

- If a technician goes to a cell containing equipment a clockwise rotation of the angle $\pi / 2$ is performed

Displacements directions

The pair $d=\left(d_{1}, d_{2}\right) \in \mathbb{Z}^{2}$ is named direction if $d_{k} \in\{-1,0,1\}^{2}, k=1,2$.

A null direction means the absence of displacement.

As the displacements are not diagonal, we have in all cases $\left|d_{1}\right|+\left|d_{2}\right| \leqslant 1$.

With $c=c_{i j}$ the displacement will be performed toward the cell $c_{i+d_{1}, j+d_{2}}$.

We denote $[d, \alpha]$ the direction $d$ with the rotation angel $\alpha\left(\alpha \in\left\{ \pm \frac{\pi}{2}, \pm \pi\right\}\right)$. for example $\left[(1,0), \frac{\pi}{2}\right]=(0,1)$ and $\left[(1,0),-\frac{\pi}{2}\right]=(0,-1)$ 


\section{Computing the technicians direction}

The displacement direction $d=\left(d_{1}, d_{2}\right)$ of a technician located in the cell $c_{i j}$, toward the cell $c_{k l}$ is given by:

$$
d=\left(d_{1}, d_{2}\right)=\left\{\begin{array}{cccc}
(1,0) & \text { si } & |k-i| ?|\ell-j| \text { et } k ? i & (\rightarrow) \\
(0,1) & \text { si } & |k-i| ?|\ell-j| \text { et } \ell ? j & (\uparrow) \\
(-1,0) & \text { si } & |k-i| ?|\ell-j| \text { et } k ? i & (\leftarrow) \\
(0,-1) & \text { si } & |k-i| ?|\ell-j| \text { et } \ell ? j & (\downarrow)
\end{array} \quad(k, \ell) \neq(i, j)\right.
$$

with, in case of equality in the conditions, we take, indifferently, any one of the directions. This direction is recalculated at each iteration to ensure the optimally of the overall path.

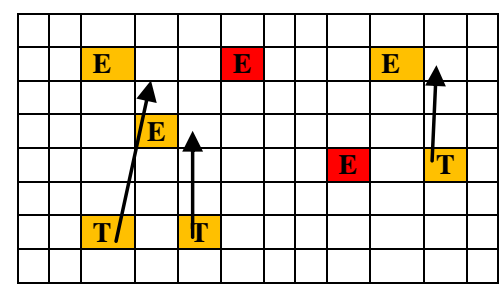

Fig.2. Tech. Displacements to target cell of the equip.

Notations: Let $c_{i j} \in \mathcal{I}$ containing an available technician $p\left(e_{t}\left(c_{i j}\right)=2\right)$ and $d$ the direction of $p$ supposed not null.

- We denote by $c_{i j}^{0}$ the neighbouring cell obtained in the direction of $d$.

- We denote by $c_{i j}^{\alpha}$ the neighbouring cell obtained by rotation of the direction $d$ by an angel $\alpha$.

- We denote by $c_{i j}^{00}$ the cell obtained in the direction $d$ with a double step. For example if $c=c_{i j}$ and $d=(1,0)$ then $c_{i j}^{00}=c_{i+2, j}$

\begin{tabular}{|l|c|c|c|c|}
\hline & & & & \\
\hline & $c^{\frac{3 \pi}{4}}$ & $c^{\frac{\pi}{2}}$ & $c^{\frac{\pi}{4}}$ & \\
\hline & $c^{\pi}$ & $\mathbf{c}$ & $c^{0}$ & $c^{00}$ \\
\hline & $c^{-\frac{3 \pi}{4}}$ & $c^{-\frac{\pi}{2}}$ & $c^{-\frac{\pi}{4}}$ & \\
\hline & & & & \\
\hline
\end{tabular}

We denote by $d_{\alpha}$ the direction of the technician located in the cell $c_{i j}^{\alpha}$ (if, however, it contains a technician)

We denote by $d_{00}$ the direction of the technician located in the cell $c_{i j}^{00}$ (if, however, it contains a technician)

The displacements rules of the technicians are illustrated through an example given in the following table:

If equipmentfails, we denote in $T[m]$ the intervention duration (time between the reporting of the failure and the restoration of the equipment)

$T[m]=\left\{\begin{array}{l}\text { elaps edtime since the intervention began } \\ \text { elapsedtime since the end of the last intervention }\end{array}\right.$

The equipment's states (which may changesat each iteration) are gathered in the following scoreboard:

Algorithm: The state of the cell $c$ at $t+1$ depends on the state of the neighbouring cells at $t$ and the technician's displacements. The $e_{t+1}\left(c_{i j}\right)$ calculation is performed by taking account the following cases: 
To simplify the notation we denote the cell $C_{i j}$ by $c$.

Case $1: \square \square \square(\square)=-1$ The cell contains the equipment $m$ its state doesn't changes :

$$
e_{t+1}(c)=e_{t}(c)
$$

Case $2: \square \square \square(\square)=2$ The cell contains an available technician $p$. If its direction $d$ is null then

$$
e_{t+1}(c)=e_{t}(c)
$$

- else :

1. If $e_{t}\left(c^{0}\right)=0$ and $e_{t}\left(c^{00}\right)=2$ or $d^{00} \neq-d_{\text {goto }} 5$

2. Rotation $d=\left[d,-\frac{\pi}{2}\right]$.

3. If rotation id performed 4 times: do $\left(e_{t+1}(c)=e_{t}(c)\right)$ then END.

4. Else goto 1

5. If $e_{t}\left(c^{-\frac{\pi}{4}}\right) \leqslant 0$ or $\left(e_{t}\left(c^{-\frac{\pi}{4}}\right)>0\right.$ and $\left.\left[d,-\frac{\pi}{2}\right]=d_{-\frac{\pi}{4}}\right): \operatorname{do}\left(e_{t+1}(c)=0\right.$ ) then END.

Case $3: \square \square \square(\square)=0$

6. Else: do $\left(e_{t+1}(c)=e_{t}(c)\right)$

1. If there's no technician in the neighbourhood: do $\left(e_{t+1}(c)=e_{t}(c)\right)$ then END.

2. Else: If no technician in the neighbourhood has its direction toward $c$ do ( $\left.e_{t+1}(c)=e_{t}(c)\right)$ END

Let $c^{0}$ the neighbouring cell containing a tech. with a direction $d_{0}$ directed to $c$.

3. If $e_{t}\left(c_{\pi}\right)>0$ and $d_{\pi}=-d_{0}: \operatorname{do}\left(e_{t+1}(c)=e_{t}(c)\right)$ then END.

4. If $e_{t}\left(c^{\frac{\pi}{2}}\right) \leq 0$ or $e_{t}\left(c^{\frac{\pi}{2}}\right)>0$ and $d_{\frac{\pi}{2}} \neq\left[d_{0},+\frac{\pi}{2}\right]: \operatorname{do}\left(e_{t+1}(c)=e_{t}\left(c^{0}\right)\right)$ then END.

5. Do $c^{0}=c^{\frac{\pi}{2}}$

6. If $c^{0}$ changes at least 3 times : goto 3 .

7. Else do $\left(e_{t+1}(c)=e_{t}(c)\right)$ then END.

\section{Simulation Results}

For the simulations we consider fixed boundary conditions. The process of the equipment's maintenance by the technicians as recorded in the assignment's schedule is performed by discrete steps in the trellis. The snapshots of the simulation are presented in figure 4 .

We suppose to simplify without losing generality that $M T B F_{j}$ and $M T T R_{j}$ are constant for all equipments. In the simulation we consider $M T B F_{j}=3$ and $M T T R_{j}=2$. The initial conditions are given in the figure 3

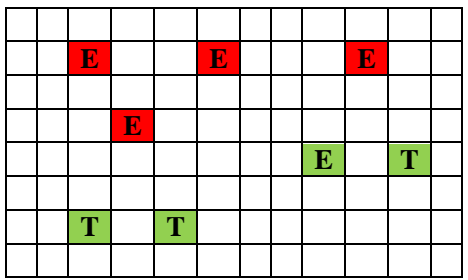

Fig.3. Initial conditions. 


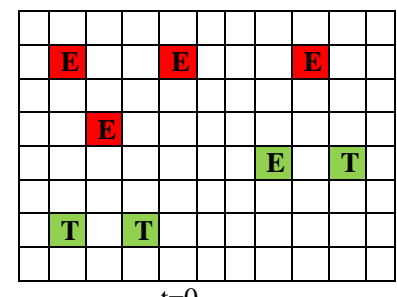

$\mathrm{t}=0$

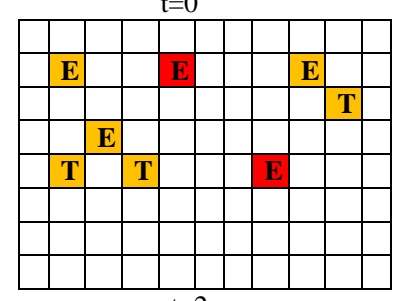

$\mathrm{t}=2$

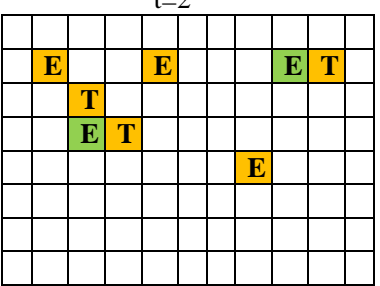

Allocation by Voronoidiagram

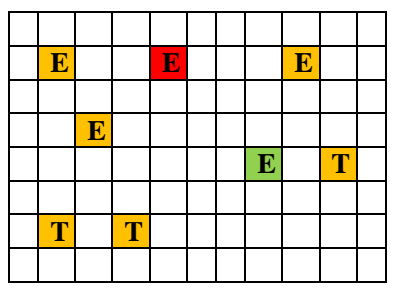

Allocation by Voronoidiagram

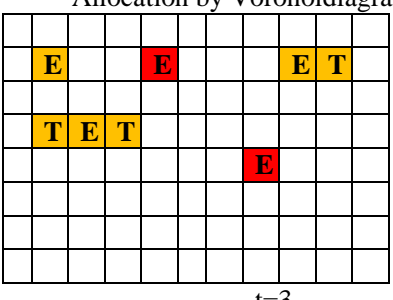

$\mathrm{t}=3$

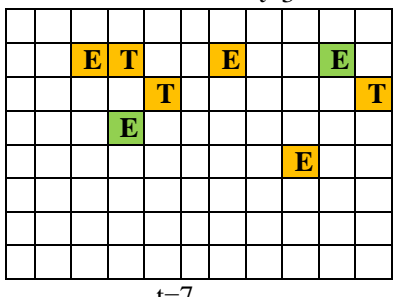

$t=7$

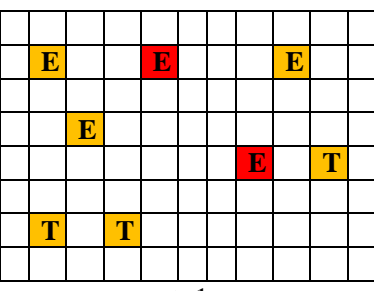

$\mathrm{t}=1$

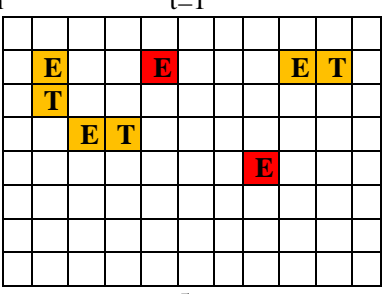

$\mathrm{t}=5$

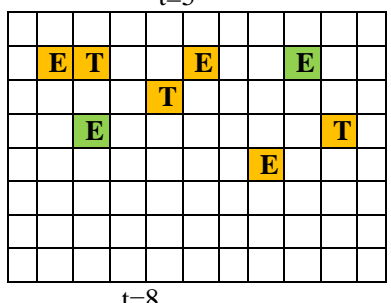

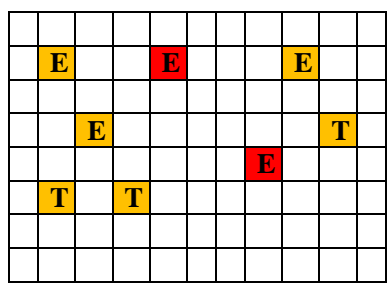

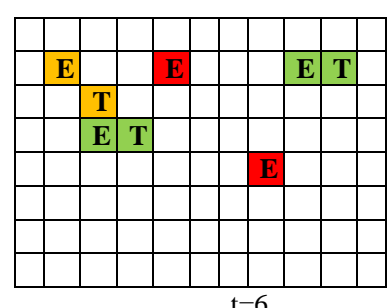

$\mathrm{t}=6$

Fig.4. Simulation results.

We see from the simulation that the use of a double Voronoi diagram for down machine and available technicians has helped to find an optimal allocation solution and the adoption of rules of displacements has guarantee a best execution of maintenance program. A new allocation scenario is performed whenever the system detects a change in the machines state (after $\mathrm{t}=6$ for example) in our simulation after $\mathrm{MTBF}_{\mathrm{j}}$ iteration. When the resource are assigned then the technicians move toward their targets (down machines), when they reach the down machine and after the $\mathrm{MTTR}_{\mathrm{i}}$ iteration the machines become operational.

\section{Conclusion}

Cellular automata have allowed us to simulate the movement of maintenance technicians based on the use of Voronoi diagram for assigning technician to down equipments.

The scenarios presented in this study will be expanded and other constraints will be added as the inclusion of unforeseen factors (random failures, unexpected absences technicians, include other states of equipments and technicians ... etc). Validation of the simulation model with real data of a company will be required once made the integration of the model with the control module of resource allocation being developed.

\section{References}

[1]. M. Bennour, D. Crestani, Using competencies in performance estimation: From the activity to the process, Computers in Industry 58 (2007) 151--163.

[2]. X. Boucher, E. Bonjour, B. Grabot, Formalization and use of competencies for industrial performance optimization: A survey, Computers in Industry 58 (2007) 98--117.

[3]. A-A. Alhouaij, Contribution à l'optimisation de la maintenance dans un contexte distribué, Ph.D. Thesis, Institut polytechnique, Grenoble, France, 2010.

[4]. C. Draghici, Modélisation et conception d'algorithmes pour la planification automatique du personnel de compagnies aériennes, Institut National de Sciences Appliquées, Toulouse, 2005.

[5]. L. Shi, C-H. Chen, A New Algorithm for Stochastic Discrete Resource Allocation Optimization. Discrete Event Dynamic Systems. Juillet 2000, Volume 10, Issue 3, pp 271-294

[6]. D. Costa, Méthodes de résolution constructives, séquentielles et évolutives pour des problèmes d'affectation sous contraintes, $\mathrm{Ph} . \mathrm{D}$. thèse, école polytechnique fédérale de Lausanne, suisse, 1995.

[7]. W-J. Gutjahr, M-S. Rauner, An ACO algorithm for a dynamic regional nurse-scheduling problem in Austria, Computers \& Operations Research, 34, pp. 642--666 (2007).

[8]. A. Bel Fekih A. El Jai (2006). Regional analysis of a class of cellular automata models, In Cellular Automata (pp. 48-57), Springer Berlin Heidelberg.

[9]. S. El Yacoubi A. El Jai (2002). Cellular automata modelling and spreadability. Mathematical and Computer Modelling, 36(9), 1059-1074.

[10]. S. Bandini, B.Chopard, M.Tomassini, Cellular Automata. 5th International Conference on Cellular Automata for Research and Industry, ACRI 2002. Geneva, Switzerland, October 2002. LNCS 2493. 